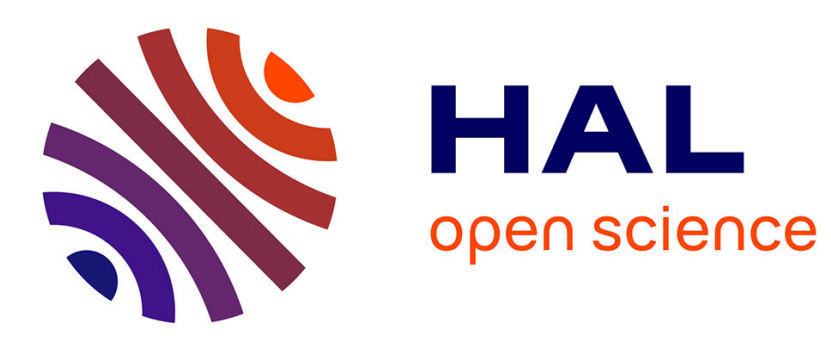

\title{
Les grandes ventes d'automne 1972 dans les forêts soumises au régime forestier
}

\author{
- [.]office National Des Forets, Paris
}

\section{To cite this version:}

- [.]office National Des Forets, Paris. Les grandes ventes d'automne 1972 dans les forêts soumises au régime forestier. Revue forestière française, 1973, 25 (2), pp.83-98. 10.4267/2042/20728 . hal03535094

\section{HAL Id: hal-03535094 \\ https://hal.science/hal-03535094}

Submitted on 19 Jan 2022

HAL is a multi-disciplinary open access archive for the deposit and dissemination of scientific research documents, whether they are published or not. The documents may come from teaching and research institutions in France or abroad, or from public or private research centers.
L'archive ouverte pluridisciplinaire HAL, est destinée au dépôt et à la diffusion de documents scientifiques de niveau recherche, publiés ou non, émanant des établissements d'enseignement et de recherche français ou étrangers, des laboratoires publics ou privés. 


\title{
LES GRANDES VENTES D'AUTOMNE 1972 DANS LES FORẼTS SOUMISES AU RÉGIME FORESTIER
}

\author{
OFFICE NATIONAL DES FORÊTS
}

Class. Oxford $756: 922$ "1971" (44)

Dans l'ensemble, les grandes ventes de bois s'ouvraient à l'automne sous des auspices favorables:

- l'activité économique en général et celles du bâtiment et de l'ameublement en particulier étaient orientées en expansion:

- la position de la France, principal producteur européen et exportateur de grumes d'essences feuillues tempérées n'était pas mauvaise compte-tenu de la raréfaction et de.la hausse des cours que connaissaient les essences tropicales depuis quelques temps. D'ailleurs, la progression régulière des indices des prix des grumes et des sciages de feuillus sur le marché intérieur depuis le début de l'année confirmait bien l'intensité de la demande:

- le marché international des résineux s'était montré très actif en 1972 et promettait de l'être encore davantage en 1973.

Toutefois quelques incertitudes subsistaient dans le domaine des résineux et dans celui des bois de trituration:

- en effet, une tempête survenue au mois d'avril avait abattu des volumes abondants de sapin-épicéa en Franche-Comté $1450000 \mathrm{~m}^{3}$ rien qu'en forêts soumises) et l'on pouvait craindre une certaine saturation du marché :

- par ailleurs, les indices des prix des grumes et des sciages de résineux (sapin-épicéa et pins) de qualité charpente et coffrage s'étaient révélés un peu flottants au cours du premier semestre de l'année:

- enfin, si l'on pouvait espérer une petite reprise des achats de bois feuillus de trituration au moins dans un certain nombre de régions, il n'en était pas de même pour les bois d'industrie résineux dont les débouchés apparaissaient quasi-inexistants, plus particulièrement encore dans le sud-est de la France. 
Toutes ces tendances se sont finalement reflétées dans les résultats des grandes ventes, qui ont été caractérisées par:

- une très forte revalorisation des grumes de feuillus ;

- une bonne fermeté, quoiqu'un peu plus irrégulière, des grumes de résineux; du chêne ;

- une timide reprise de l'écoulement des feuillus de trituration à l'exception

- une mévente quasi-totale des bois d'industrie résineux.

A des quantités offertes équivalentes en feuillus et un peu inférieures en résineux $(-5 \%)$ à celles de l'automne dernier, ont correspondu des volumes vendus accrus en feuillus $(+8 \%)$ venant compenser des volumes vendus moindres en résineux $(-5 \%)$.

La demande s'est donc montrée pressante en matière de feuillus, assez soutenue en ce qui concerne le sapin-épicéa (compte tenu des importants chablis de FrancheComté qui avaient été commercialisés au printemps), peu active pour les pins (en particulier les pins maritimes et les pins divers).

En regard des autres années (voir Annexe III), la proportion des feuillus invendus paraît particulièrement faible tandis que celle des résineux équivalente à celle de l'automne 1971 dépasse sensiblement la norme.

Les invendus portent essentiellement sur les bois médiocres ou de petites dimensions (surtout résineux), sur les taillis et sur les lots d'exploitation difficile. Comme l'an dernier, ils sont surtout localisés en Provence-Côte d'Azur, Languedoc-Roussillon, Rhône-Alpes et Aquitaine.

Tableau $n^{\circ} 1 \quad$ Volume mis en vente et volume vendu (en $1000 \mathrm{~m}^{3}$ )

\begin{tabular}{|l|c|c|c|c|c|c|}
\hline & \multicolumn{2}{|c|}{ Volume mis en vente } & \multicolumn{2}{c|}{ Volume vendu } & \multicolumn{2}{c|}{$\%$ invendu } \\
\cline { 2 - 7 } & 1971 & 1972 & 1971 & 1972 & 1971 & 1972 \\
\hline Feuillus & 2635 & 2670 & 2215 & 2400 & $16 \%$ & $10 \%$ \\
Résineux & 2795 & 2630 & 2160 & 2030 & $23 \%$ & $23 \%$ \\
Taillis + Houppiers & 1925 & 1895 & 1520 & 1590 & $21 \%$ & $16 \%$ \\
TOTAL & $\mathbf{7 3 5 5}$ & $\mathbf{7 1 9 5}$ & $\mathbf{5 8 9 5}$ & $\mathbf{6 0 2 0}$ & $\mathbf{2 0 \%}$ & $\mathbf{1 6 \%}$ \\
\hline
\end{tabular}

En se chiffrant à près de 350 millions de $F$, les recettes sont en très sensible progression sur celles des dernières années. Cette augmentation est encore plus marquée dans les forêts domaniales (197,2 millions de $F$ contre 159,4 à l'automne 1971) qui comprennent une proportion plus importante de feuillus de qualité, que dans les forêts des collectivités publiques $(152,2$ millions de $F$ contre 143,4$)$ à prédominance de résineux.

Comme les volumes vendus n'ont que très peu augmenté globalement $(+2 \%)$, cet accroissement des recettes traduit une montée certaine des prix. Le prix moyen, calculé sans tenir compte des taillis et des houppiers, accuse une hausse de 


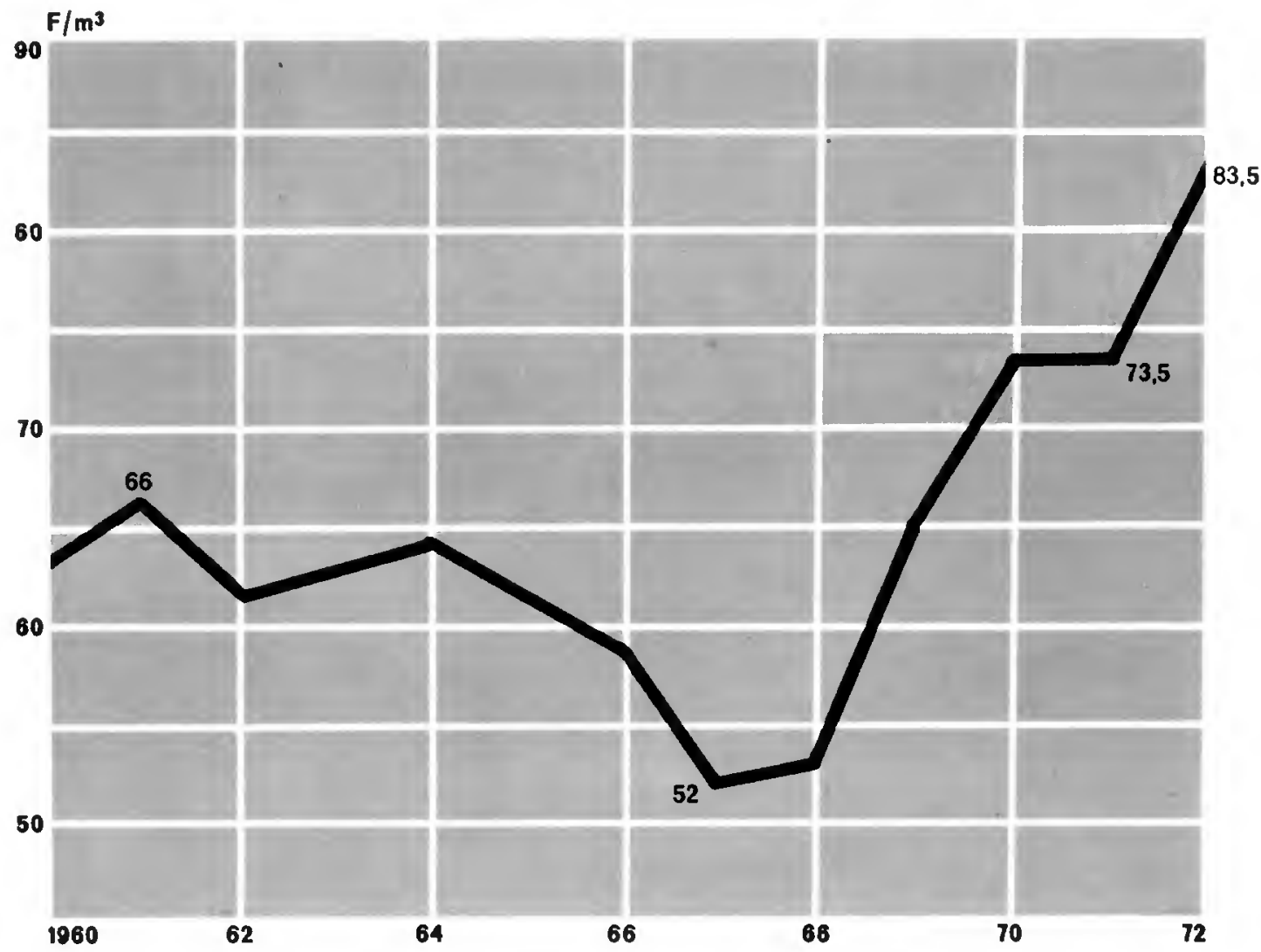

Graphique n* 1

TOUTES ESSENCES

Evolution du prix moyen aux Grendes Ventes

(Taillis et houppiers exclus - Taxe forfaiteire comprise)

près de $14 \%$ sur l'automne dernier. Cependant compte tenu de la diminution des invendus en feuillus et du meilleur écoulement des bois mitraillés, cette hausse moyenne est sans doute un peu sous-estimée.

Par ailleurs, elle recouvre une évolution très différente selon qu'il s'agit de feuillus ou de résineux.

\section{LE CHÊNE}

En 1972, le chêne a tenu la vedette comme en témoigne le très faible pourcentage des invendus et la forte hausse des cours.

Les quantités offertes et les quantités absorbées par le marché ont été supérieures à celles des années précédentes : les pourcentages d'invendus ont même été les plus bas parmi ceux enregistrés au cours des dix dernières années, au moins en ce qui concerne les chênes de gros et moyens diamètres. Par contre, une certaine 
mévente règne toujours sur les chênes de petites dimensions, la légère reprise des achats de bois de trituration s'étant en priorité portée sur le hêtre et le charme.

\section{CHÊNE}

Diagramme $n^{\circ} 1$

Volume mis en vente et volume vendu

(en $1000 \mathrm{~m}^{3}$ )
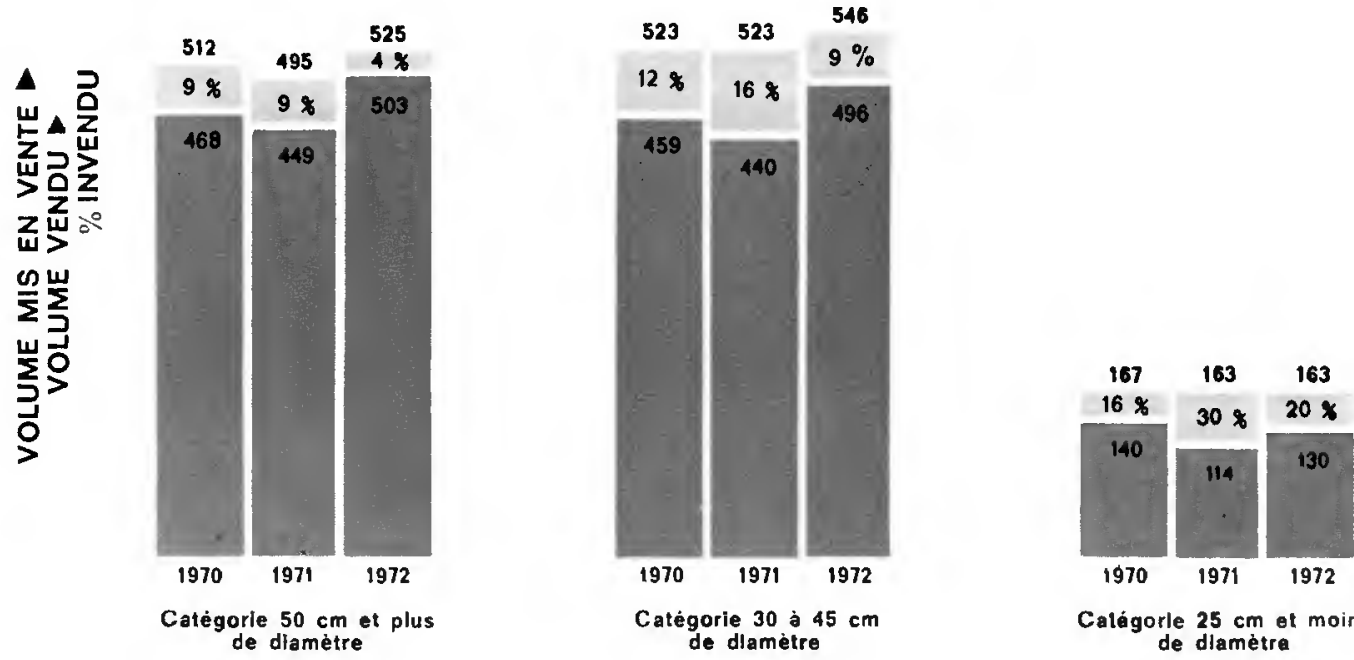

Calágorle $25 \mathrm{~cm}$ et moins de dlamètra

Après des années de quasi stagnation, surtout si l'on tient compte de l'érosion de la monnaie, le prix moyen du chêne a accusé une augmentation de plus de $25 \%$ entre 1971 et 1972.

La hausse de $30 \%$ constatée en moyenne sur les chênes de $50 \mathrm{~cm}$ et plus de diamètre traduit bien la vive concurrence dont ont été l'objet les belles qualités de chêne : les qualités menuiserie, ébénisterie et merrains ont été particulièrement recherchées puisqu'elles ont bien souvent enregistré des hausses encore plus prononcées, pouvant atteindre 40 à $50 \%$; pour les bois de tranchage, la hausse semble avoir été moins forte sur les grands crûs (Auvergne, Centre...), qui bénéficiaient déjà de cours très élevés, que sur les qualités traditionnellement moins exceptionnelles (Bourgogne, Champagne).

Dans le tableau $n^{\circ} 2$, la faible amplitude de la hausse dans le Loir-et-Cher s'explique pour une différence de qualité : à qualités égales, elle ressortirait à environ

Tableau $n^{\circ} 2$

Chêne de $50 \mathrm{~cm}$ et plus de diemètre

Principaux départements producteurs - Grandes Ventes 1972

\begin{tabular}{|l|c|c|c|c|}
\hline Départements & $\begin{array}{c}\text { Prix moyen } \\
1972(1)\end{array}$ & $\begin{array}{c}\text { Variation } \\
1972-1971\end{array}$ & $\begin{array}{c}\text { Volume vendu } \\
\left(\mathrm{m}^{3}\right)\end{array}$ & $\begin{array}{c}\text { Proportion } \\
\text { d'invendus }\end{array}$ \\
\hline Allier & 250 & $+22 \%$ & 22900 & $0 \%$ \\
Loir-et-Cher & 318 & $+8 \%$ & 10000 & $0 \%$ \\
Indre & 357 & $+26 \%$ & 13600 & $1 \%$ \\
Orne & 261 & $+25 \%$ & 8500 & $5 \%$ \\
Nièvre & 222 & $+27 \%$ & 24900 & $4 \%$ \\
Côte d'Or & 155 & $+22 \%$ & 40600 & $1 \%$ \\
Haute-Marne & 193 & $+48 \%$ & 27100 & $4 \%$ \\
\hline
\end{tabular}

(1) Taxe forfaitaire comprise 


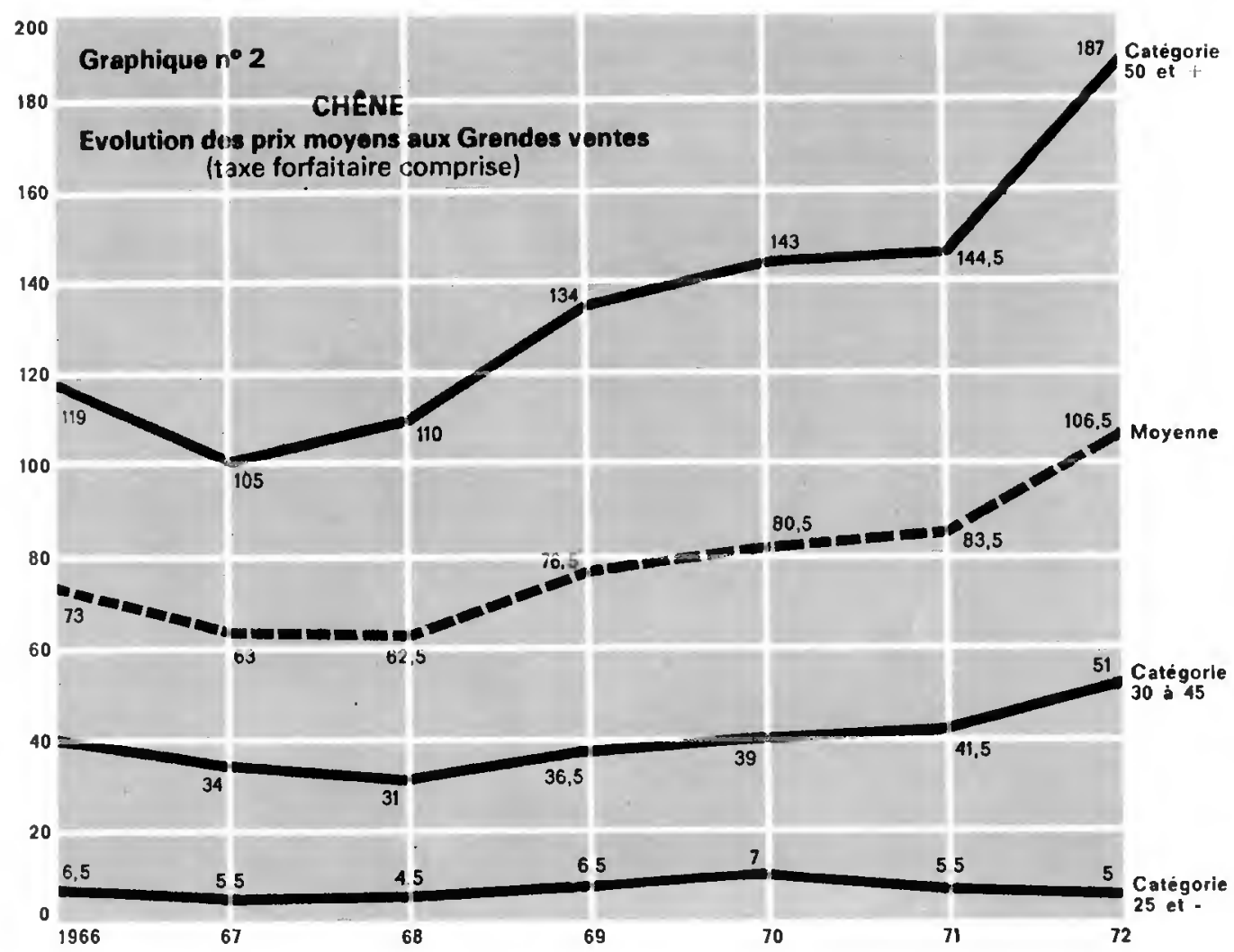

$30 \%$. Par ailleurs, on note l'inexistence ou la quasi-inexistence d'invendus, ce qui a dans l'ensemble pour conséquence de tempérer légèrement la hausse.

Les diamètres moyens (30 à $45 \mathrm{~cm}$ ) ont connu également des augmentations très importantes, parfois même supérieures à celles des gros diamètres dans un certain nombre de régions (voir tableau $n^{\circ} 3$ ). Elles s'expliquent, entre autres, par la vigueur du marché des palettes. On doit remarquer également la très faible importance des invendus, ce qui est un peu inhabituel pour cette catégorie.

Tableau n' 3 Chêne de 30 à $45 \mathrm{~cm}$ de diemètre

Principaux départements producteurs - Grandes Ventes 1972

\begin{tabular}{|l|c|c|c|c|}
\hline Départements & $\begin{array}{c}\text { Prix moyen } \\
1972(1)\end{array}$ & $\begin{array}{c}\text { Variation } \\
1972-1971\end{array}$ & $\begin{array}{c}\text { Volume vendu } \\
\left(\mathrm{m}^{3}\right)\end{array}$ & $\begin{array}{c}\text { Proportion } \\
\text { d'invendus }\end{array}$ \\
\hline Allier & 60 & $+12 \%$ & 42900 & $1 \%$ \\
Loir-et-Cher & 106 & $+43 \%$ & 11700 & $0 \%$ \\
Indre & 88 & $+26 \%$ & 11100 & $2 \%$ \\
Orne & 74 & $+35 \%$ & 20500 & $6 \%$ \\
Nièvre & 42 & $+18 \%$ & 24400 & $4 \%$ \\
Cóte-d'Or & 40 & $+15 \%$ & 28300 & $10 \%$ \\
Haute-Marne & 43 & $+28 \%$ & 18300 & $14 \%$ \\
\hline
\end{tabular}

(1) Taxe forfaitaire comprise 
Le prix moven du chêne de $25 \mathrm{~cm}$ et moins de diamètre marque une baisse de $10 \%$ qui vient se joindre à des invendus relativement abondants, bien qu'en diminution sur ceux de l'an dernier : en effet, les arbres les plus gros de cette catégorie ont bénéficié d'une certaine demande avec la progression de la technique qui permet de scier des arbres de dimensions de plus en plus petites.

\section{LE HÊTRE}

Une grande animation a également caractérisé le marché du hêtre au cours de la période des grandes ventes, ce qui a permis de commercialiser des quantités plus importantes qu'à l'automne 1971, alors que les volumes offerts étaient plutôt moindres.

\section{Diagramme $n^{\circ} 2$}
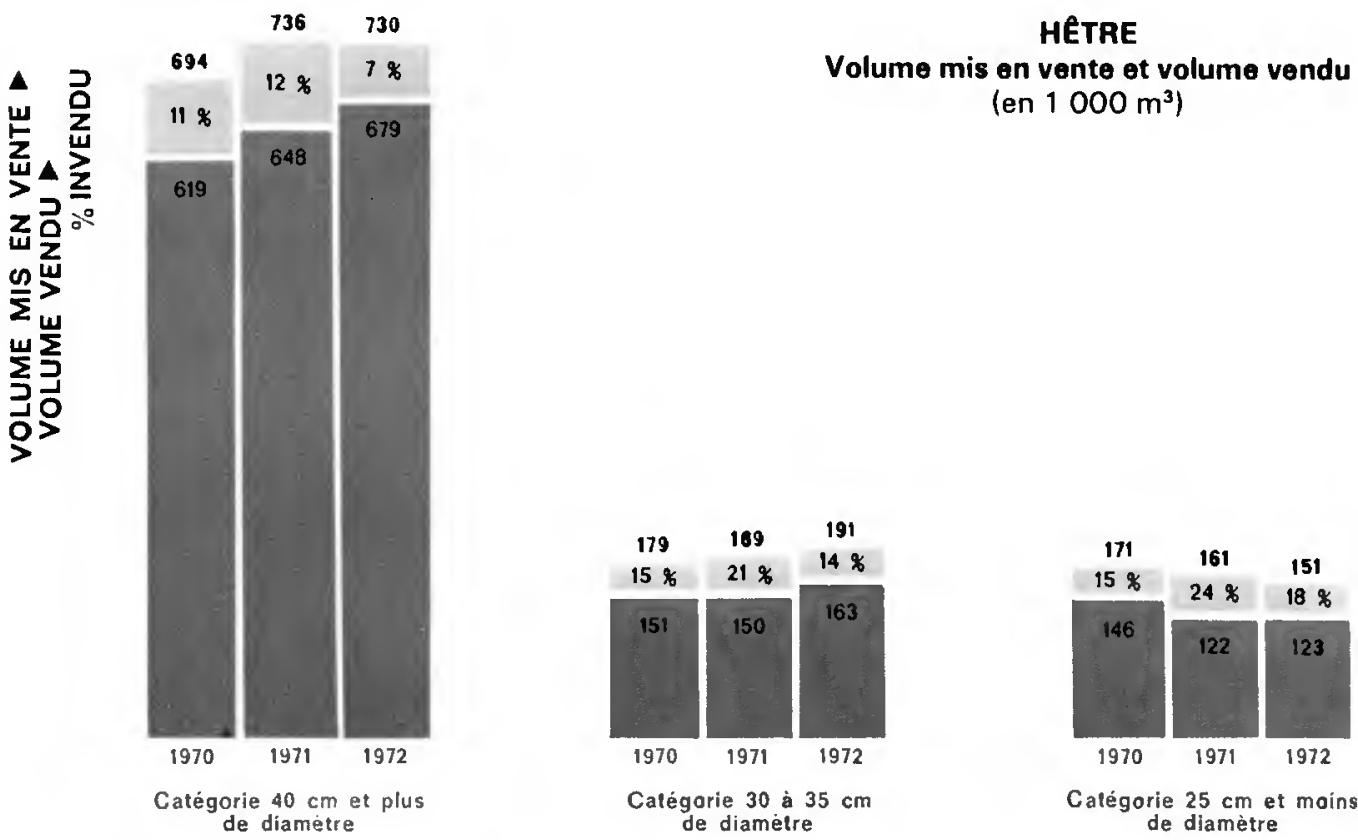

Catégorie $25 \mathrm{~cm}$ et maíns de diamètre

L'expansion actuelle des industries, du déroulage, des palettes et de la caisserie ainsi que l'existence d'une très forte demande extérieure explique les vives hausses enregistrées par le hêtre : $+31 \%$ pour les gros diamètres, $+22 \%$ pour les movens et $+15 \%$ pour les petits qui ont bénéficié de la reprise des achats de bois de trituration dans un certain nombre de régions et de la préférence accordée au hêtre sur les autres essences en période de pléthore.

Toutefois, si la hausse moyenne du hêtre s'élève à $30 \%$, ce sont essentiellement les très fortes hausses constatées en Normandie, en Picardie et dans les PyrénéesAtlantiques, sous l'effet d'une très forte demande à l'exportation (Angleterre, Espagnel, qui ont pesé sur le prix moyen national; dans les autres régions, les hausses avoisinent $15 \%$. 
Graphique $n^{\circ} 3$

HÊTRE

Evolution des prix moyens aux grendes ventes

(taxe forfaitaire comprise)

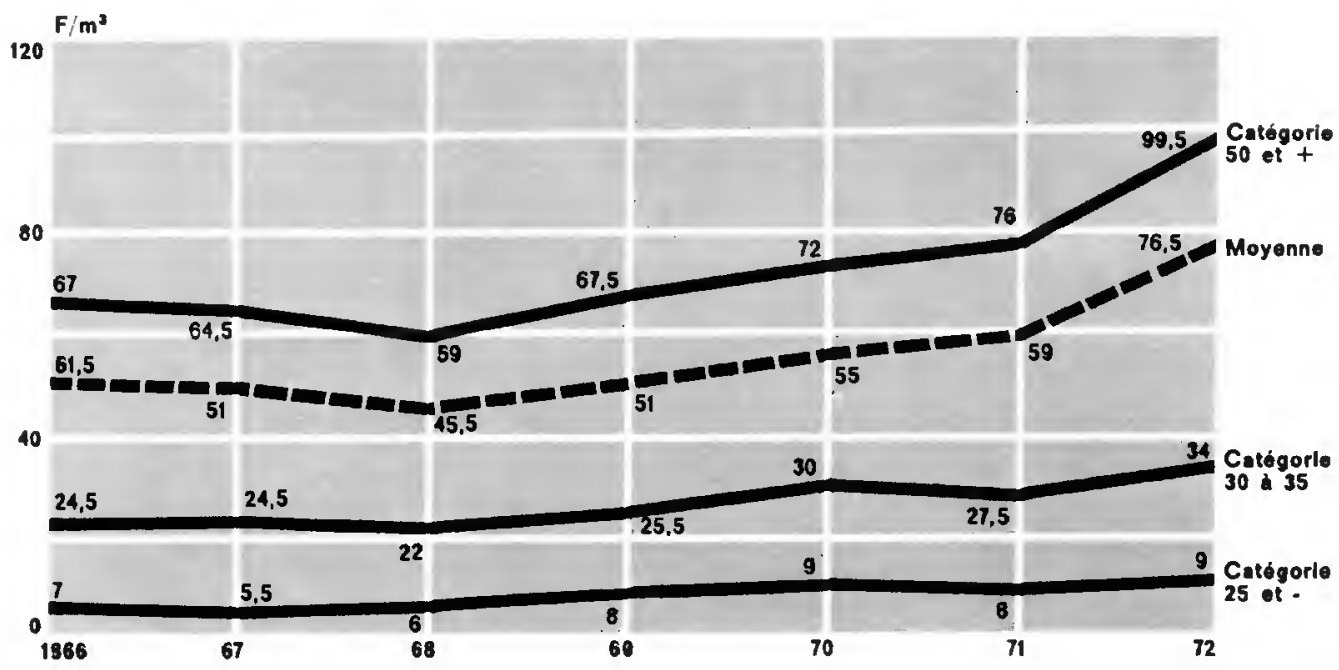

Tableau $n^{\circ} 4$

Hétre de $40 \mathrm{~cm}$ et plus de diamétre

Principaux départements producteurs - Grandes ventes 1972

\begin{tabular}{|l|c|c|c|c|}
\hline Départements & $\begin{array}{c}\text { Prix moven } \\
1972(1)\end{array}$ & $\begin{array}{c}\text { Variation } \\
1972-1971\end{array}$ & $\begin{array}{c}\text { Volume vendu } \\
\left(\mathrm{m}^{3}\right)\end{array}$ & $\begin{array}{c}\text { Proportion } \\
\text { d'invendus }\end{array}$ \\
\hline Seine-Maritime & 149 & $+52 \%$ & 74300 & $0 \%$ \\
Oise & 91 & $+68 \%$ & 38700 & $1 \%$ \\
Aisne & 87 & $+33 \%$ & 31300 & $0 \%$ \\
Haute-Marne & 125 & $+16 \%$ & 31900 & $3 \%$ \\
Meuse & 86 & $+9 \%$ & 64700 & $8 \%$ \\
Prrénées-Atlant. & 150 & $+45 \%$ & 28400 & $1 \%$ \\
\hline
\end{tabular}

(1) Taxe forfaitaire comprise

\section{LES FEUILLUS DIVERS}

Tout en accusant une amélioration sensible, la proportion des invendus demeure encore assez importante, ce qui est vraisemblablement dû au malaise qui caractérise encore le marché de la trituration.

Tableau $n^{\circ} 5$

Feuillus divers

Volume mis en vente et volume vendu

$\left(1000 \mathrm{~m}^{3}\right)$

\begin{tabular}{|c|c|c|c|}
\hline & Volume mis en vente & Volume vendu & $\%$ invendu \\
\hline 1970 & 341 & 290 & $15 \%$ \\
1971 & 356 & 278 & $22 \%$ \\
1972 & 351 & 295 & $16 \%$ \\
\hline
\end{tabular}


Etant donné l'hétérogénéïté des produits que recouvre cette rubrique, il est très difficile d'en suivre l'évolution des cours; la tendance a varié selon la destination des essences : si les bois de trituration n'ont pas fait l'objet d'une forte concurrence, il n'en a pas été de même pour le frêne et les arbres fruitiers qui enregistrent des hausses très prononcées.

\section{LE SAPIN ET L'ÉPICÉA}

Si l'offre apparaît moins conséquente aux ventes d'automne 1972, ce n'est dû qu'aux très volumineux chablis de Franche-Comté qui ont été commercialisés dès le printemps et qui ont amené à réduire l'offre de bois frais dans cette région à l'automne. Moyennant quoi le marché n'en a pas été trop perturbé puisque le pourcentage des invendus ne s'est pas montré supérieur à celui de l'an dernier. Ces invendus sont d'ailleurs particulièrement peu importants en Franche-Comté,comme le montre le tableau $n^{\circ} 6$. Ils sont en fait essentiellement déterminés par les difficultés d'exploitation et sont donc surtout localisés dans les régions de haute montagne, les régions savoyardes en particulier, et concernent en premier lieu l'épicéa.

Diagramme $n^{\circ} 3$

\section{SAPIN ET ÉPICÉA \\ Volume mis en vente et volume vendu (en $1000 \mathrm{~m}^{3}$ )}
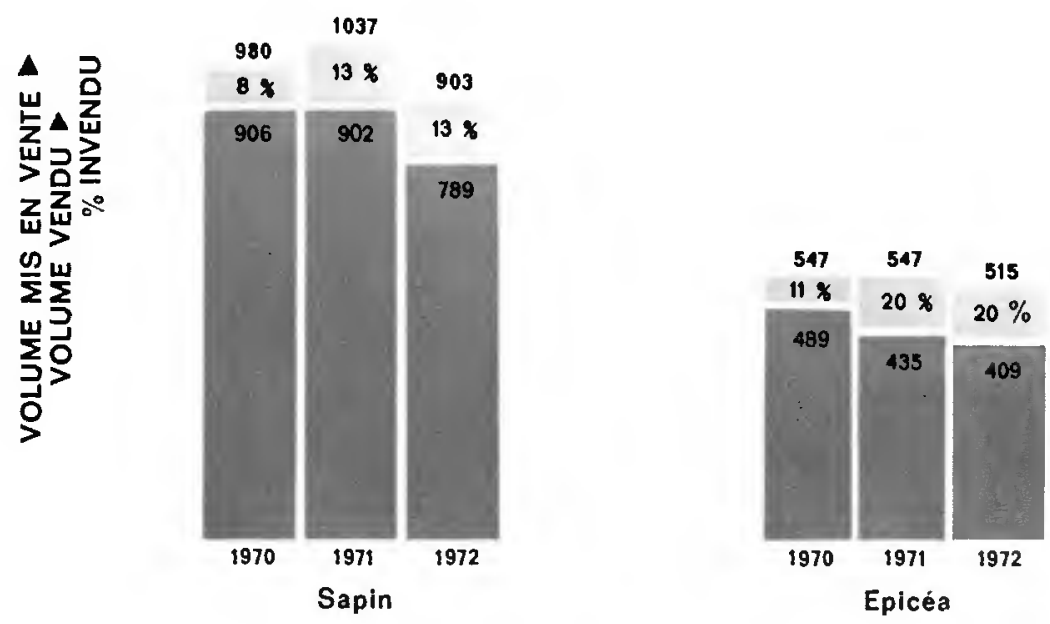

En moyenne, les prix du sapin et de l'épicéa sont restés stationnaires à un ou deux points près par rapport à l'automne 1971 et ne rejoignent même pas tout à fait le niveau de 1970.

Cette stabilité d'ensemble recouvre en fait des disparités régionales importantes, allant d'une hausse sensible dans les Vosges à une baisse non moins accentuée dans les Pyrénées, en passant par une hausse modérée ou même la stabilité dans les autres départements producteurs. Ces divers mouvements aboutissent ainsi à une relative uniformisation des prix dans les différentes régions (voir tableau $n^{0} 6$ ). 
Graphique $n^{\circ} 4$

\section{SAPIN ET EPICEA}

Evolution des prix moyens aux grandes ventes

(taxe forfaitaire comprise)

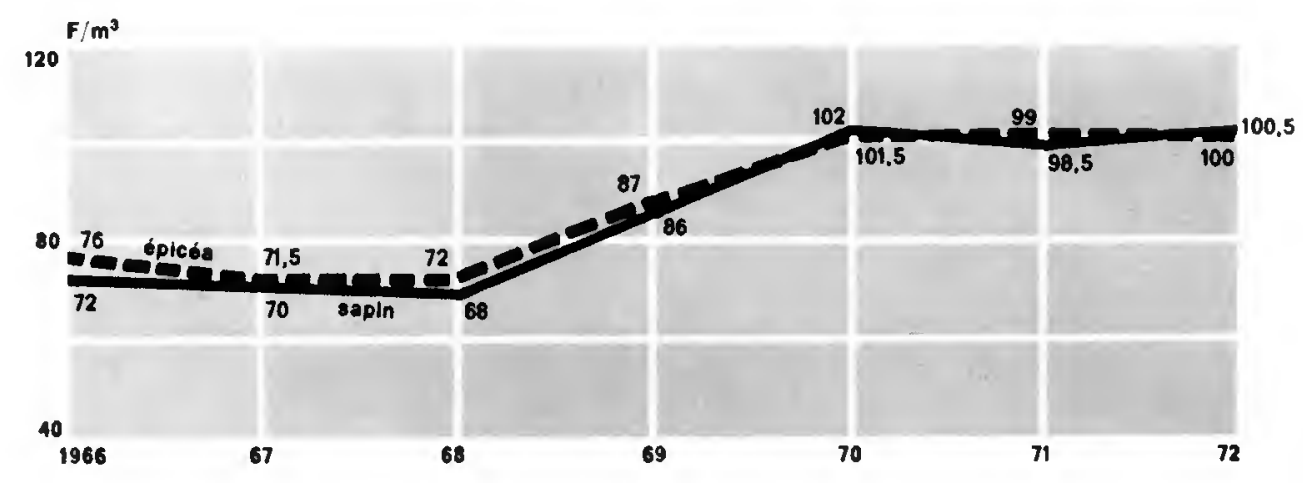

Tableau $n^{\circ} 6$

Sapin et épicéa de plus de $25 \mathrm{~cm}$ de diemétre

Principaux départements producteurs - Grandes ventes 1972

\begin{tabular}{|l|c|c|c|c|c|}
\hline Départements & Essences & $\begin{array}{c}\text { Prix moyen } \\
1972(1)\end{array}$ & $\begin{array}{c}\text { Variation } \\
1972-1971\end{array}$ & $\begin{array}{c}\text { Volume vendu } \\
\left(\mathrm{m}^{3}\right)\end{array}$ & $\begin{array}{c}\text { Proportion } \\
\text { d'invendus }^{\mathbf{3}}\end{array}$ \\
\hline Vosges & Sapin & 104 & $+14 \%$ & 247600 & $2 \%$ \\
& Epicéa & 122 & $+16 \%$ & 43400 & $2 \%$ \\
Doubs & Sapin & 118 & $+4 \%$ & 92200 & $0 \%$ \\
& Epicea & 126 & $+3 \%$ & 45800 & $1 \%$ \\
Jura & Sapin & 126 & $+8 \%$ & 5100 & $5 \%$ \\
Ain & Epicea & 126 & $+5 \%$ & 6500 & $1 \%$ \\
Haute-Savoie & Sapin & 108 & $+6 \%$ & 66200 & $9 \%$ \\
& Epicea & 113 & $+13 \%$ & 15300 & $5 \%$ \\
Savoie & Sapin & 93 & 0 & 23300 & $16 \%$ \\
Aude & Epicea & 112 & $+4 \%$ & 61600 & $17 \%$ \\
Hautes- & Sapin & 81 & $+6 \%$ & 38200 & $28 \%$ \\
Pyrénées & Epicéa & 84 & $+1 \%$ & 64100 & $41 \%$ \\
& Sapin & 126 & $-20 \%$ & 21600 & $19 \%$ \\
\hline
\end{tabular}

(1) Taxe forfaitaire comprise

\section{LE PIN SYLVESTRE}

Bien que l'on note une nette amélioration de la commercialisation par rapport à 1971, les invendus restent cependant volumineux.

Les $2 / 3$ de ces invendus sont localisés en Provence Côte-d'Azur et en RhôneAlpes et sont liés à la crise des bois de trituration résineux, particulièrement grave dans le Sud-Est de la France. 
Tableau $n^{\circ} 7 \quad$ Pin sylvestre - Volume mis en vente et volume vendu $\left(1000 \mathrm{~m}^{3}\right)$

\begin{tabular}{|c|c|c|c|}
\hline & Volume mis en vente & Volume vendu & \% invendu \\
\hline 1970 & 504 & 447 & $11 \%$ \\
1971 & 537 & 386 & $28 \%$ \\
1972 & 565 & 441 & $22 \%$ \\
\hline
\end{tabular}

Cette amélioration de la situation s'est non seulement traduite en quantités, mais aussi en prix puisque ceux-ci accusent une hausse moyenne de $6 \%$. Cette hausse n'a d'ailleurs concerné que les gros bois; les prix des petits bois ont au contraire continué à fléchir.

L'étude de l'évolution des prix du pin sylvestre sur plusieurs années montre une certaine similitude avec celle du sapin, mais les fluctuations en sont beaucoup plus accusées.

II en est ainsi très vraisemblablement parce qu'il s'agit d'une essence de complément.

\section{Graphique $n^{\circ} 5$}

PIN SYLVESTRE

Evolution des prix moyens eux grandes ventes (taxe forfaitaire comprise)

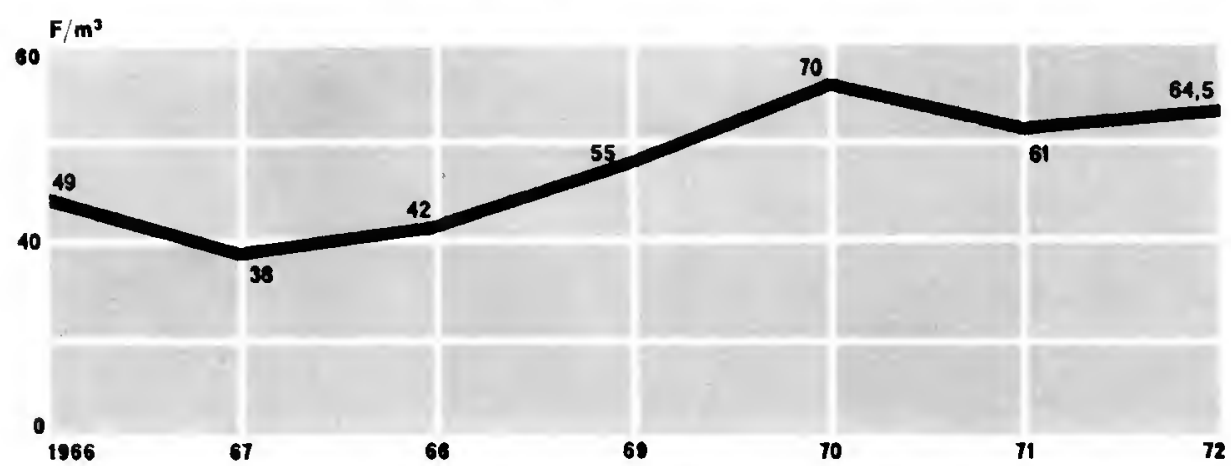

Si quelques points de hausse ont bien été observés dans les plus gros départements producteurs comme le Loiret, l'Orne ou les Vosges, on note à l'inverse quelques points de baisse dans d'autres départements comme la Seine et Marne, la SeineMaritime ou le Puy-de-Dóme. En fait, même dans ces départements les belles qualités ont été orientées en hausse; le tassement n'a concerné que les bois de qualité plus moyenne.

Tableau $n^{\circ} 8$

Pin sylvestre de $25 \mathrm{~cm}$ et plus de diamètre Principaux départements producteurs - Grandes ventes 1972

\begin{tabular}{|l|c|c|c|c|}
\hline Départements & $\begin{array}{c}\text { Prix } \\
1972(1)\end{array}$ & $\begin{array}{c}\text { Variation } \\
1972-1971\end{array}$ & $\begin{array}{c}\text { Volume vendu } \\
\left(\mathrm{m}^{3}\right)\end{array}$ & $\begin{array}{c}\text { Proportion } \\
\text { d'invendus }\end{array}$ \\
\hline Loiret & 70 & $+6 \%$ & 68400 & $2 \%$ \\
Seine-et-Marne & 65 & $-6 \%$ & 10600 & $0 \%$ \\
Orne & 95 & $+10 \%$ & 36900 & $2 \%$ \\
Seine-Maritime & 85 & $-4 \%$ & 28800 & $10 \%$ \\
Puy-de-Dôme & 69 & $-8 \%$ & 13600 & $19 \%$ \\
Vosges & 50 & $+15 \%$ & 39100 & $6 \%$ \\
\hline
\end{tabular}

(1) Taxe forfaitaire comprise 


\section{LE PIN MARITIME}

Les invendus sont encore plus importants qu'à l'automne 1971 et témoignent du peu d'empressement pour cette essence cette année, lié d'ailleurs, pour une grande partie, à la crise des bois d'industrie.

Tableau $n^{\circ} 9 \quad$ Pin maritime - Volume mis en vente et volume vendu

$\left(1000 \mathrm{~m}^{3}\right)$

\begin{tabular}{|c|c|c|c|}
\hline & Volume mis en vente & Volume vendu & $\%$ invendu \\
\hline 1970 & 315 & 284 & $10 \%$ \\
1971 & 353 & 274 & $22 \%$ \\
1972 & 355 & 244 & $31 \%$ \\
\hline
\end{tabular}

La hausse moyenne relevée (+ $6 \%$ ) est trompeuse : compte-tenu de l'accroissement des invendus et donc de la commercialisation, à l'automne 1972, des qualités les meilleures, les cours se sont montrés plutôt stables, voire en léger tassement.

Graphique $n^{\circ} 6$

PIN MARITIME

Evolution des prix moyens aux grandes ventes (taxe forfaitaire comprise)

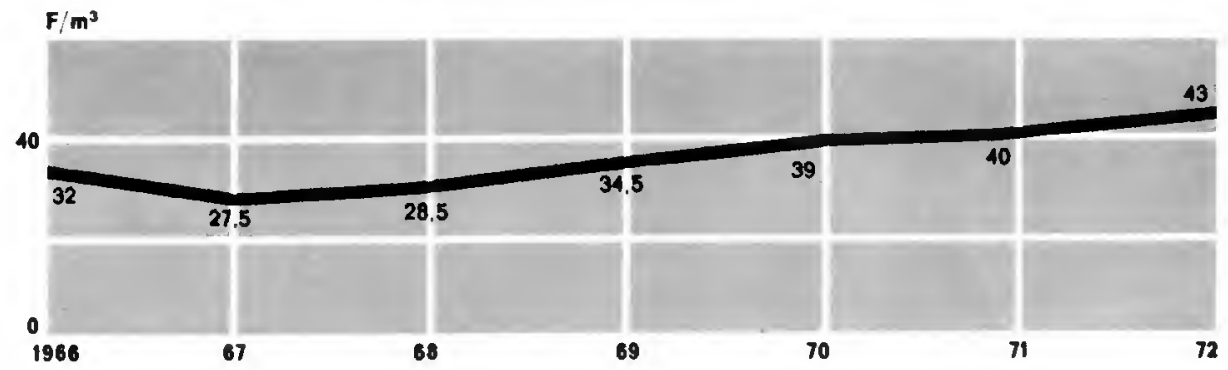

Le marché s'est montré encore plus déprimé en Gironde que dans les Landes où la qualité offerte cette année était plutôt meilleure.

Tableau $n^{\circ} 10 \quad$ Pin maritime de $25 \mathrm{~cm}$ et plus de diamètre

Principaux départements producteurs - Grandes ventes 1972

\begin{tabular}{|l|c|c|c|c|}
\hline Départements & $\begin{array}{c}\text { Prix moyen } \\
1972(1)\end{array}$ & $\begin{array}{c}\text { Variation } \\
1972-1971\end{array}$ & $\begin{array}{c}\text { Volume vendu } \\
\left(\mathrm{m}^{3}\right)\end{array}$ & $\begin{array}{c}\text { Proportion } \\
\text { d'invendus }\end{array}$ \\
\hline $\begin{array}{l}\text { Gironde } \\
\text { Landes }\end{array}$ & 39 & $-5 \%$ & 35300 & $54 \%$ \\
121400 & $23 \%$ \\
\hline
\end{tabular}

(1) Taxe forfaitaire comprise

\section{LES RÉSINEUX DIVERS}

Comme en 1971, près de la moitié de l'offre est restée pour compte : en effet, une grande part de ces bois sont destinés à la trituration et n'ont pas trouvé à s'écouler en raison de la persistance de la crise du marché des bois d'industrie de résineux: tel a été le cas en particulier du pin noir de trituration des Alpes qui n'a été l'objet d'aucune demande, ni française, ni italienne. 
Tableau no 11 Résineux divers - Volume mis en vente et volume vendu $\left(1000 \mathrm{~m}^{3}\right)$

\begin{tabular}{|c|c|c|c|}
\hline & Volume mis en vente & Volume vendu & $\%$ invendu \\
\hline 1970 & 317 & 237 & $25 \%$ \\
1971 & 319 & 162 & $49 \%$ \\
1972 & 291 & 151 & $48 \%$ \\
\hline
\end{tabular}

En raison de la faiblesse de la demande, les prix ont dans l'ensemble été orientés en baisse ; toutefois, des distorsions apparaissent selon les essences ou les régions: les prix du mélèze se sont montrés fermes, ceux du pin noir de Lozère en hausse, mais ceci s'explique pour deux raisons : d'une part, on offrait en Lozère une forte proportion d'arbres à poteaux dont la demande est grande et d'autre part. les chablis avaient fortement pesé sur les prix de l'automne 1971 qui avaient accusé une forte baisse par rapport a 1970 (- $32 \%)$ : les prix de 1972 ne font qu'opérer un léger redressement.

Ailleurs, les prix du pin noir sont en baisse, de même que ceux du pin à crochets et même du pin laricio qui avaient, il est vrai, connu de fortes hausses au cours des années précédentes.

Tableau $\mathrm{n}^{\circ} 12$ Résineux divers de $25 \mathrm{~cm}$ et plus de diemètre

Principaux départements producteurs - Grandes ventes 1972

\begin{tabular}{|l|c|c|c|c|}
\hline \multicolumn{1}{|c|}{ Départements } & $\begin{array}{c}\text { Prix moyen } \\
1972(1)\end{array}$ & $\begin{array}{c}\text { Variation } \\
1972-1971\end{array}$ & $\begin{array}{c}\text { Volume vendu } \\
\left(\mathrm{m}^{3}\right)\end{array}$ & $\begin{array}{c}\text { Proportion } \\
\text { d'invendus }\end{array}$ \\
\hline $\begin{array}{l}\text { Hautes-Alpes (2) } \\
\text { Alpes de Haute- }\end{array}$ & 62 & $-8 \%$ & 24300 & $46 \%$ \\
Provence (3) & 35 & $-15 \%$ & 12600 & $58 \%$ \\
Drôme (4) & 16 & $-15 \%$ & 6700 & $53 \%$ \\
Lozère (5) & 41 & $+16 \%$ & 16800 & $16 \%$ \\
Pyrénées- & 38 & $-25 \%$ & 4700 & $77 \%$ \\
Orientales (6) & 90 & $-37 \%$ & 7300 & $65 \%$ \\
Corse (7) & & & & \\
\hline
\end{tabular}

(1) Taxe forfaitaire comprise

(2) Essentiellement mélèze et pin noir

(3) Essentiellement pin noir, accessoirement pin à crochets et mélèze
(4) Uniquement pin noir

(5) Essentiellement pin noir et pin à crochets

(6) Uniquement pin à crochets

(7) Uniquement pin laricio

\section{LES PETITS BOIS}

A l'exception des coupes comprenant des poteaux de ligne, la commercialisation des petits bois' résineux s'avère très difficile, voire impossible (pins du Sud-Est).

En ce qui concerne les feuillus, l'écoulement est plus ou moins facile selon qu'on se trouve dans une zone d'approvisionnement d'une usine de trituration ou non. C'est ainsi que l'écoulement des petits bois de hêtre et de charme n'a guère posé de problème en Picardie, Normandie, Poitou, Haute-Marne, Haute-Saône..., mais les industries utilisatrices se désintéressent très largement du chêne et abandonnent tout achat dans certaines régions (Ardennes, Bourgogne, Alpes, Massif Central, Bretagne). 
Située dans un cadre annuel, la hausse des prix des bois sur pied, plus particulièrement des feuillus peut paraître considérable. Mais replacée dans un contexte général à plus long terme, elle apparaît comme une revalorisation normale : cette hausse est moins forte que celle des produits semi-transformés (grumes et sciages), comme on peut le voir dans le tableau ci-après.

Evolution des prix de 1965 a 1972

\begin{tabular}{|l|c|c|c|}
\hline & sur pieds* & grumes** & sciages** \\
\hline Chêne & $+45 \%$ & +70 d $80 \%$ & +50 d $55 \%$ \\
(dont 50 et + ) & $(+60 \%)$ & & $+60 \%$ \\
Hêtre & $+45 \%$ & +60 d $65 \%$ & +40 d $45 \%$ \\
Sapin-Epicéa & $+30 \%$ & +40 d $50 \%$ & +35 d $40 \%$ \\
Pin sylvestre & $+25 \%$ & +15 d $20 \%$ & +30 d $35 \%$ \\
Pin maritime & $+20 \%$ & $+50 \%$ & \\
Toutes essences et & $+35 \%$ & & \\
toutes catégories & & & \\
\hline
\end{tabular}

Moyenne nationale des prix de gros et de détail : $+35 \%$

- Source : O.N.F.

* Source : Moniteur des Travaux publics

Rappelons en outre, qu'au cours de la période précédant celle-ci, c'est-à-dire de 1960 à 1965, les prix des bois sur pied ont baissé de manière sensible, tandis que le coût de la vie progressait à une allure moyenne de $4 \%$ par an; c'est dire que la revalorisation des prix de 1972 par rapport à 1960 ne compense pas, pour le propriétaire forestier, la perte de pouvoir d'achat de la monnaie.

OFFICE NATIONAL DES FORETTS

Département commercial

4. avenue de Saint-Mandé 75570 PARIS CEDEX 12 
(taxe forfaitaire comprise)

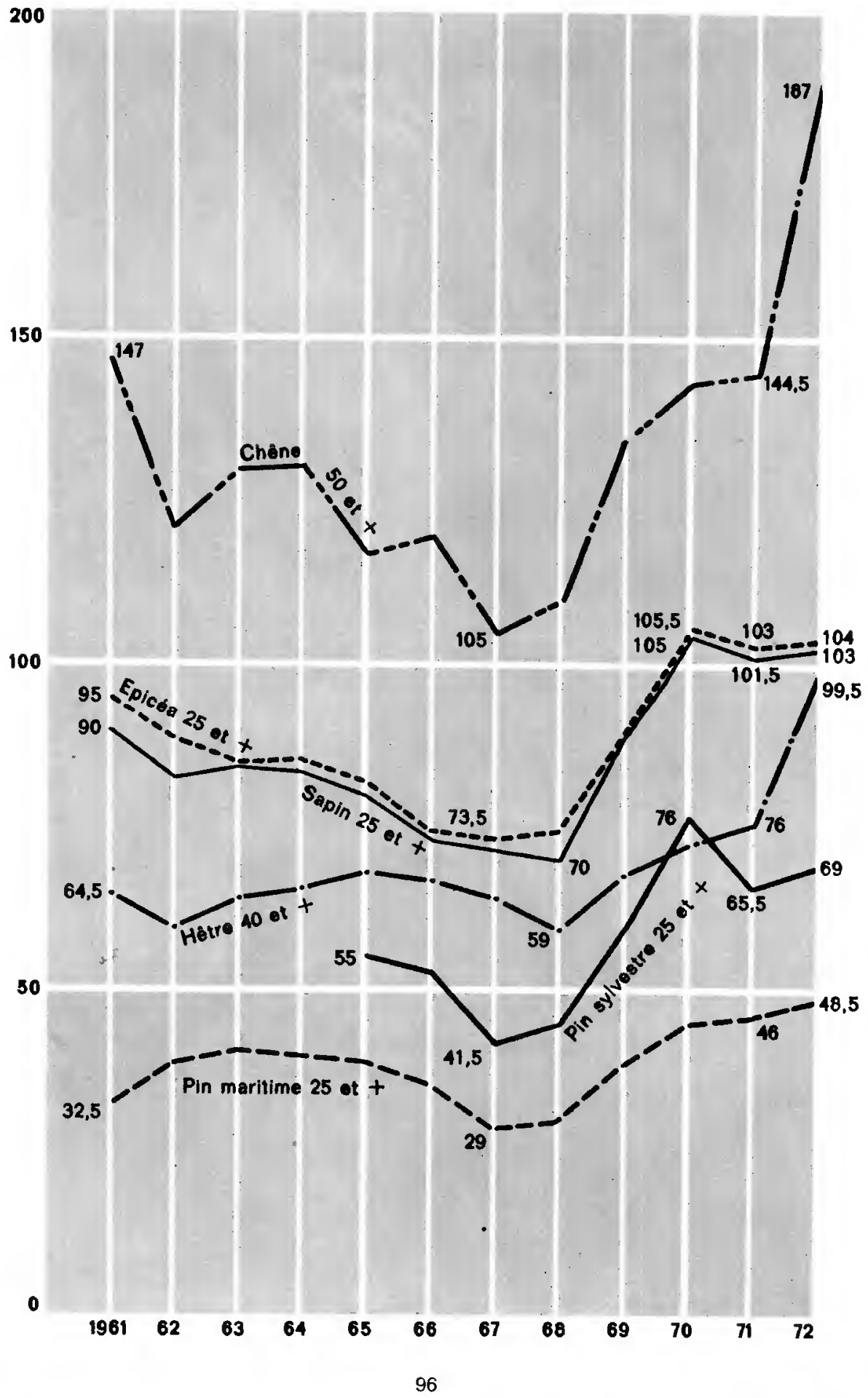


GRANDES VENTES 1972

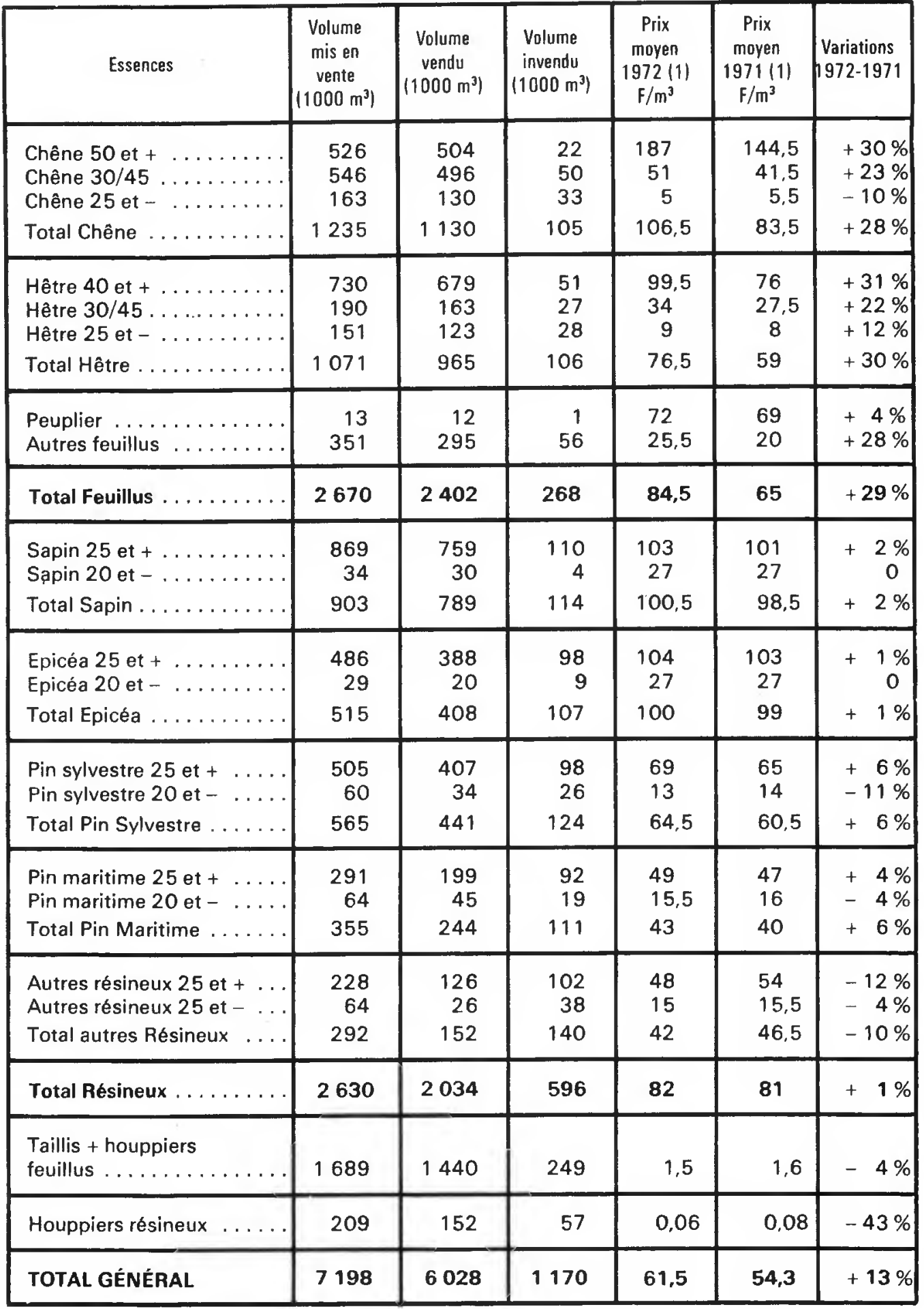

(1) Taxe forfaitaire comprise 
ANNEXE III

RÉSULTATS COMPARÉS

DES GRANDES VENTES DEPUIS 10 ANS

\begin{tabular}{|c|c|c|c|c|c|c|}
\hline Années & Catégories (1) & $\begin{array}{l}\text { Volume } \\
\text { mis en } \\
\text { vente } \\
\left(1000 \mathrm{~m}^{3}\right)\end{array}$ & $\begin{array}{c}\text { Volume } \\
\text { vendu } \\
\left(1000 \mathrm{~m}^{3}\right)\end{array}$ & $\begin{array}{l}\text { Volume } \\
\text { invendu } \\
\left(1000 \mathrm{~m}^{3}\right)\end{array}$ & $\begin{array}{c}\% \\
\text { invendu }\end{array}$ & $\begin{array}{c}\text { Recettes } \\
\text { (millions } \\
\text { de F } \\
\text { courants) } \\
\quad(2)\end{array}$ \\
\hline 1962 & $\begin{array}{l}\text { Feuillus } \ldots \ldots \ldots \\
\text { Résineux . . . . . } \\
\text { Total . . . . }\end{array}$ & $\begin{array}{l}4188 \\
2401 \\
6589\end{array}$ & $\begin{array}{l}3363 \\
2098 \\
5461\end{array}$ & $\begin{array}{r}825 \\
303 \\
1128\end{array}$ & $\begin{array}{l}20 \% \\
13 \% \\
17 \%\end{array}$ & 216,1 \\
\hline 1963 & $\begin{array}{l}\text { Feuillus . . . . . . } \\
\text { Résineux . . . . } \\
\text { Total . . . . }\end{array}$ & $\begin{array}{l}4014 \\
2477 \\
6491\end{array}$ & $\begin{array}{l}3357 \\
2124 \\
5481\end{array}$ & $\begin{array}{r}657 \\
353 \\
1010\end{array}$ & $\begin{array}{l}16 \% \\
14 \% \\
16 \%\end{array}$ & 225,8 \\
\hline 1964 & $\begin{array}{l}\text { Feuillus } \ldots \ldots \ldots \\
\text { Résineux } \ldots \ldots \ldots \\
\text { Total } \ldots \ldots \ldots\end{array}$ & $\begin{array}{l}3970 \\
2473 \\
6443\end{array}$ & $\begin{array}{l}3330 \\
2137 \\
5467\end{array}$ & $\begin{array}{l}640 \\
336 \\
976\end{array}$ & $\begin{array}{l}16 \% \\
14 \% \\
15 \%\end{array}$ & 227,8 \\
\hline 1965 & 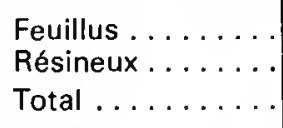 & $\begin{array}{l}4181 \\
2767 \\
6948\end{array}$ & $\begin{array}{l}3433 \\
2340 \\
5773\end{array}$ & $\begin{array}{r}748 \\
427 \\
1175\end{array}$ & $\begin{array}{l}18 \% \\
15 \% \\
17 \%\end{array}$ & 247,4 \\
\hline 1966 & $\begin{array}{l}\text { Feuillus ........ } \\
\text { Résineux ....... } \\
\text { Total ......... }\end{array}$ & $\begin{array}{l}4104 \\
2847 \\
6951\end{array}$ & $\begin{array}{ll}3 & 177 \\
2 & 131 \\
5 & 308\end{array}$ & $\begin{array}{r}927 \\
716 \\
1643\end{array}$ & $\begin{array}{l}23 \% \\
25 \% \\
24 \%\end{array}$ & 217,3 \\
\hline 1967 & $\begin{array}{l}\text { Feuillus } \ldots \ldots \ldots \\
\text { Résineux . . . . . } \\
\text { Total } \ldots \ldots\end{array}$ & $\begin{array}{l}3826 \\
2335 \\
6161\end{array}$ & $\begin{array}{l}2691 \\
1631 \\
4322\end{array}$ & $\begin{array}{r}1135 \\
704 \\
1839\end{array}$ & $\begin{array}{l}29 \% \\
30 \% \\
29 \%\end{array}$ & 158,8 \\
\hline 1968 & $\begin{array}{l}\text { feuillus } \ldots \ldots \ldots \\
\text { Résineux . . . . . } \\
\text { Total . . . . }\end{array}$ & $\begin{array}{l}4109 \\
2614 \\
6723\end{array}$ & $\begin{array}{l}3201 \\
2130 \\
5331\end{array}$ & $\begin{array}{r}908 \\
484 \\
1392\end{array}$ & $\begin{array}{l}22 \% \\
18 \% \\
21 \%\end{array}$ & 202,5 \\
\hline 1969 & $\begin{array}{l}\text { Feuillus . . . . . } \\
\text { Résineux . . . . . } \\
\text { Total . . . . . }\end{array}$ & $\begin{array}{l}4133 \\
2952 \\
7085\end{array}$ & $\begin{array}{l}3696 \\
2657 \\
6353\end{array}$ & $\begin{array}{l}437 \\
295 \\
732\end{array}$ & $\begin{array}{l}11 \% \\
10 \% \\
10 \%\end{array}$ & 304,3 \\
\hline 1970 & $\begin{array}{l}\text { Feuillus . . . . . . } \\
\text { Résineux . . . . . } \\
\text { Total . . . . }\end{array}$ & $\begin{array}{l}4321 \\
2884 \\
7205\end{array}$ & $\begin{array}{l}3718 \\
2553 \\
6271\end{array}$ & $\begin{array}{l}603 \\
331 \\
934\end{array}$ & $\begin{array}{l}14 \% \\
11 \% \\
13 \%\end{array}$ & 342,1 \\
\hline 1971 & $\begin{array}{l}\text { Feuillus . . . . . . } \\
\text { Résineux . . . . . } \\
\text { Total . . . . . }\end{array}$ & $\begin{array}{l}4336 \\
3034 \\
7370\end{array}$ & $\begin{array}{l}3577 \\
2331 \\
5908\end{array}$ & $\begin{array}{r}759 \\
703 \\
1462\end{array}$ & $\begin{array}{l}17 \% \\
23 \% \\
20 \%\end{array}$ & 321,1 \\
\hline 1972 & $\begin{array}{l}\text { Feuillus } \ldots \ldots \ldots \\
\text { Résineux . . . . . } \\
\text { Total . . . . . }\end{array}$ & $\begin{array}{l}4360 \\
2840 \\
7200\end{array}$ & $\begin{array}{ll}3 & 843 \\
2 & 186 \\
6 & 029\end{array}$ & $\begin{array}{r}517 \\
654 \\
1171\end{array}$ & $\begin{array}{l}12 \% \\
23 \% \\
16 \%\end{array}$ & 370.4 \\
\hline
\end{tabular}

(1) Les volumes de feuillus et résineux s'entendent toutes catégories comprises

(2) Les recettes s'entendent charges et taxe forfaitaire incluses. 